\title{
Fabrication of low stray light holographic gratings for space applications
}

\author{
Reinhard Steiner, Alexander Pesch, Lars H. Erdmann, Matthias Burkhardt; Alexandre Gatto, Carl \\ Zeiss Jena GmbH (Germany); \\ Robert Wipf, Torsten Diehl ${ }^{1}$; Carl Zeiss Microscopy (Germany); \\ H. J. P. Vink, B. G. van den Bosch, TNO (Netherlands); (Germany)
}

\begin{abstract}
The main challenges of fabricating diffraction gratings for use in earth monitoring spectrometers are given by the requirements for low stray light, high diffraction efficiency and a low polarization sensitivity. Furthermore the use in space also requires a high environmental stability of these gratings.

We found that holography in combination with ion beam plasma etching provides a way to obtain monolithic, robust fused silica gratings which are able to meet the above mentioned requirements for space applications. Holography accompanied by plasma etching allows the fabrication of a wide range of different grating profiles to optimize the efficiency including the polarization behavior according to a wealth of applications. Typical profile shapes feasible are blazed gratings, sinusoidal profiles and binary profiles and this allows to tailor the efficiency and polarization requirements exactly to the spectral range of the special application. Holographic gratings can be fabricated on plane and also on curved substrates as core components of imaging spectrometers.

In this paper we present our grating fabrication flow for the example of plane blazed gratings and we relate the efficiency and stray light measurement results to certain steps of the process. The holographic setup was optimized to minimize stray light and ghosting recorded by the photoresist during the exposure. Low wave front deviations require the use of highly accurate grating substrates and high precision optics in the holographic exposure.
\end{abstract}

Keywords: grating, custom design grating, holography, stray light, efficiency, space application.

\section{INTRODUCTION}

The very challenging specifications mandatory in the development of special gratings for space applications request such a high degree of technology that only a few players in the word are able to engineer them. Efficiency, low scatter level and polarization sensitivity as well as environmental robustness are the key parameters for each grating used in space applications. Zeiss has developed a core technology that masters all these diverse requirements. Here, we present recent results obtained so far in the frame of the TROPOMI project.

TROPOMI (TROPOspheric Monitoring Instrument) is a spectrometric system covering the bands in the UV, the VIS, the NIR and SWIR bands (see Figure 1 for some details). As a space borne nadir viewing instrument it will be part of the GMES Sentinel 5 Precursor mission by ESA. Within this mission, the atmospheric composition will be measured spatially resolved. Under observation are the constituents ozone, nitrogen dioxide, sulfur dioxide, carbon monoxide, methane and formaldehyde as well as aerosol properties. This data can then be used for climate and air quality applications.

\footnotetext{
${ }^{1}$ To whom all correspondence should be addressed: torsten.diehl@zeiss.com; phone +49-3641-64-3273; www.zeiss.de/gratings
}

Imaging Spectrometry XVIII, edited by Pantazis Mouroulis, Thomas S. Pagano, Proc. of SPIE Vol. 8870, 88700H - @ 2013 SPIE · CCC code: 0277-786X/13/\$18 - doi: 10.1117/12.2025269 


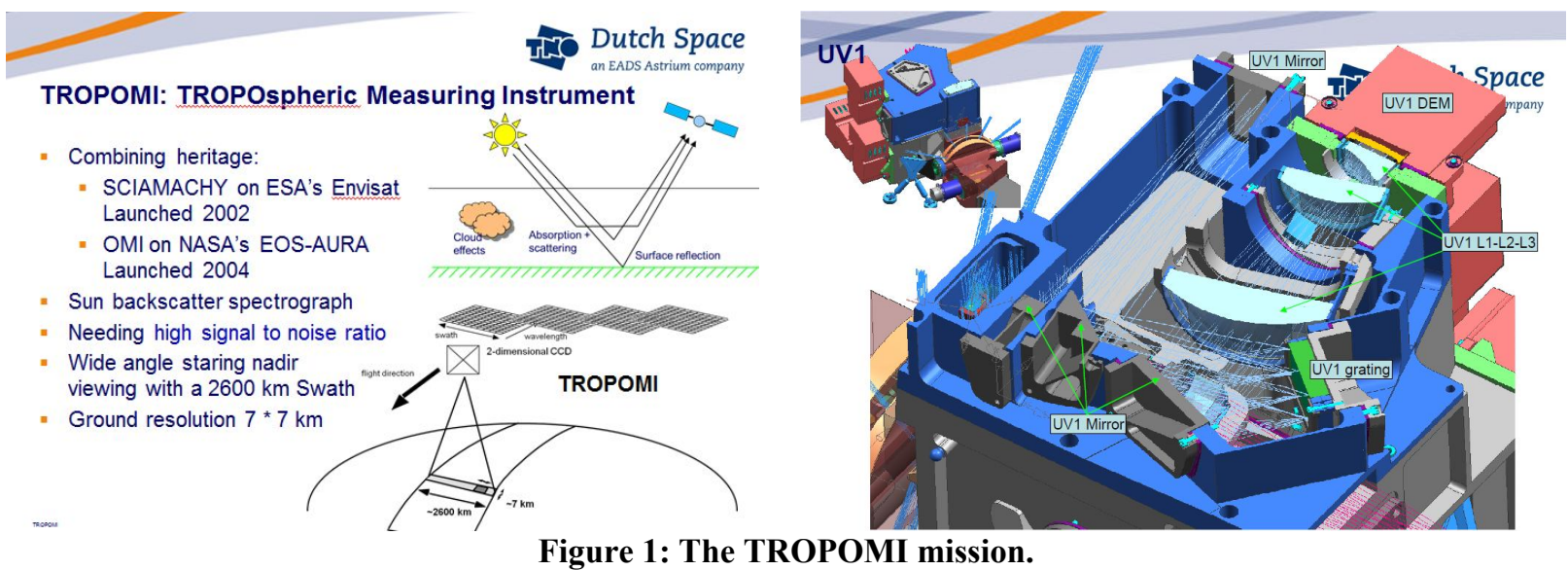

In this context, the Sentinel 5 Precursor will extent currently recorded data provided by the OMI (Ozone Monitoring Instrument, NASA) and the SCIAMACHY (SCanning Imaging Absorption spectroMeter for Atmospheric CartograpHY, ESA). In this way, the Sentinel 5 Precursor provides the link between the current scientific missions and the Sentinel 4 and Sentinel 5 missions. [1]

The TROPOMI project originates from an initiative in the Netherlands and is managed by the Netherlands Space Office (NSO). Included in this project are KNMI and SRON as Principal Investigators as well as Dutch Space and TNO. In this group TNO is developing the spectrometers. Two of these spectrometers are the UV2VIS and NIR spectrometers, covering the wavelength regimes between $310 \mathrm{~nm}$ to $500 \mathrm{~nm}$ and between $675 \mathrm{~nm}$ and $775 \mathrm{~nm}$ respectively. For these spectrometers ZEISS was the partner for the development of the diffraction gratings of these spectrometers (see Figure 2 for the optical appearance of one TROPOMI grating made by Zeiss). In this project, ZEISS and TNO worked closely together using the long expertise of both partners in technology and metrology for optical systems. In the following, the technological process and the resulting performance of the UV2VIS grating for TROPOMI will be presented.

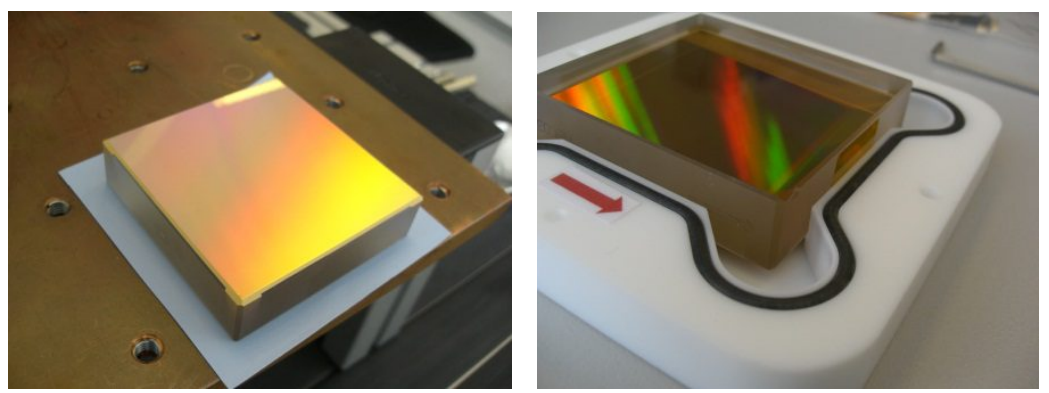

Figure 2: Photographs of one of the TROPOMI flight gratings.

\section{FABRICATION OF HOLOGRAPHIC LOW STRAYLIGHT GRATINGS}

Many years of experience in the development and manufacture of diffraction gratings make Zeiss the right address for high quality diffractive optics [5-12]. The technical basis of production are holographic exposure systems and ultra-highprecision ruling engines which offer multiple possibilities of modification. The benefits of holographically produced gratings are their high diffraction efficiency, even with high groove frequencies, and their very low stray light. Holographic procedures also allow the generation of symmetric and asymmetric groove profiles, such as blaze profiles, directly obtained after exposure and development. Mechanically ruled Zeiss gratings distinguish themselves by particular uniform groove spacing, resulting in low stray light. This ensures that the systems (such as spectrometric instruments) perform with high detection sensitivity even for low intensity signals and provide increased measurement accuracy over a broad wavelength range. Since a long time Zeiss produces customized gratings meeting critical demands on the basis 
of a broad collection of core technologies. Our main core technologies are optical design, fabrication of customized substrates, specialized coatings, high technology patterning steps, dry etching processes as well as testing and metrology capacities. Figure 3 shows a brief summary of these core competences and capabilities of Zeiss.

Optical Design

- Rigorous Modeling

- Wave-Optical Engineering

Metrology and Testing

- AFM

- SEM

- Confocal Microscopy

- Phase Shift Interferometer

- Optical Characterization

- Thermal Vacuum Testing

- Certification
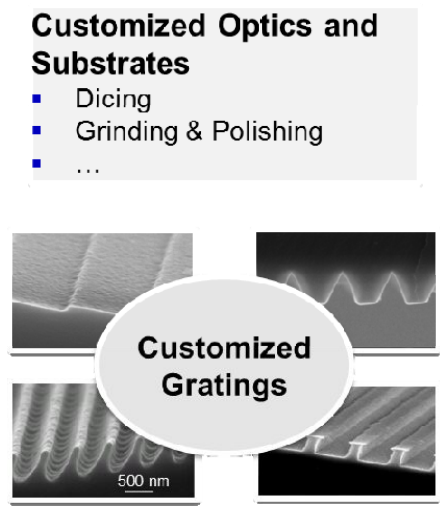

Coatings

- Design

- Metallic Coating

- Reflective Coatings

- Anti-Reflective Coatings
Patterning

- Interference Lithography

- Mechanical Ruling

- Grey Scale Lithography

Dry Etching Processes

- IBE

- RIBE

Replication

- Several Processes

- Free Choice of Substrate Material

Figure 3: Zeiss core technologies available for use in grating manufacturing.

The experience gathered over decades in the production of special optical systems is of vital importance in the polishing of mirrors as well as grating substrates for special applications. Ultra-fine polishing techniques provide all grating substrates with optimum properties for the subsequent structuring processes. Substrate geometries from plane over spherical to aspherical or even free form surfaces with the required micro-roughness of $\leq 0.3 \mathrm{~nm}$ rms are possible.

One particular ability is the direct recording of blaze profiles, besides recording symmetric profiles (see

Figure 4 and Figure 5). Directly recorded blaze gratings have significant advantages when compared to mechanically ruled or blazed gratings made by ion beam etching of sinusoidal gratings. The biggest advantage is reduced surface roughness, which decreases grating scatter and improves the detection limits of the system.

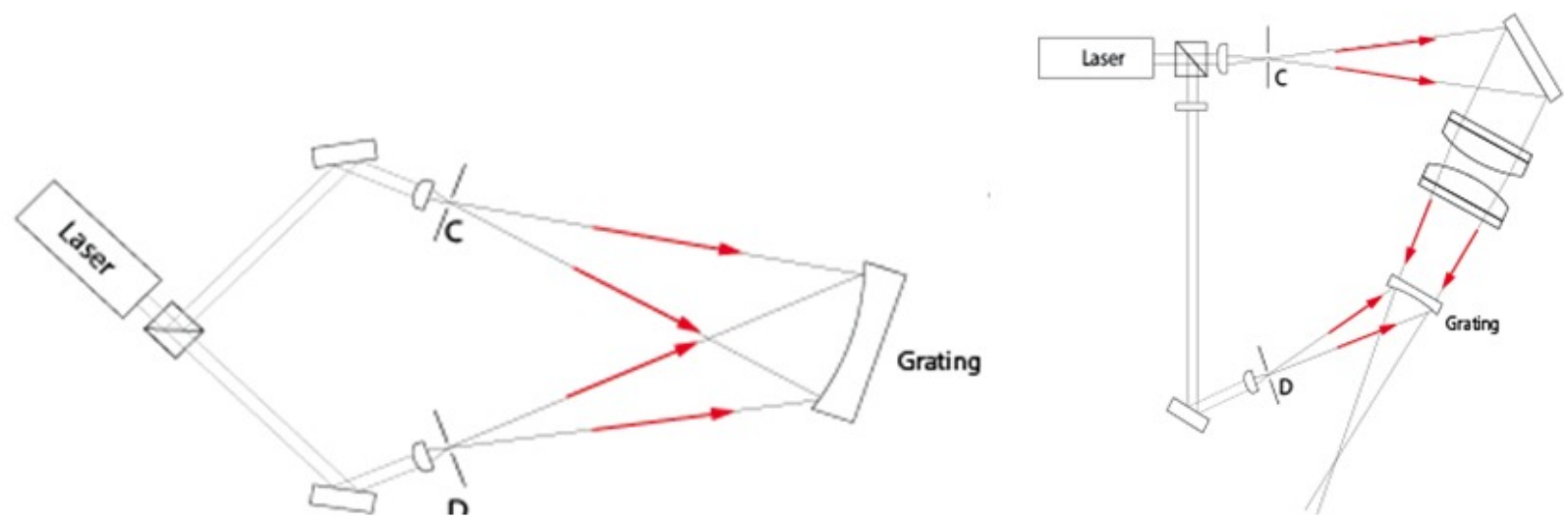

Figure 4: Sketch of basic setups used to get symmetric (left figure) and blazed (right figure) grating profiles. 


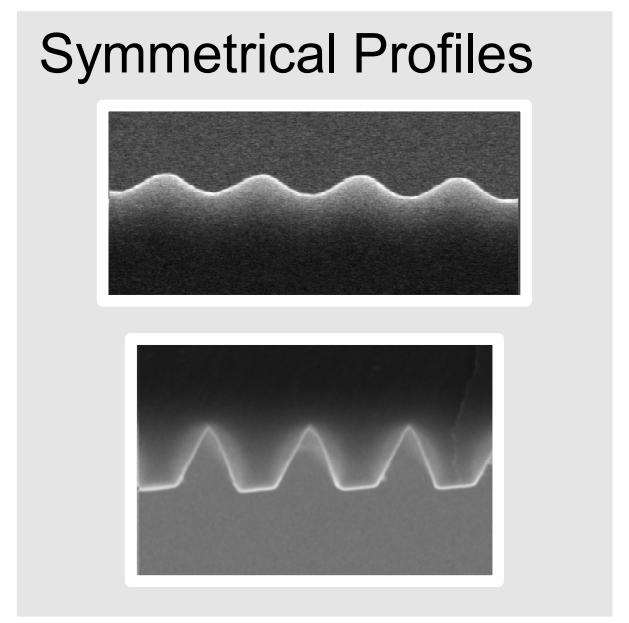

\section{Blaze Profiles}

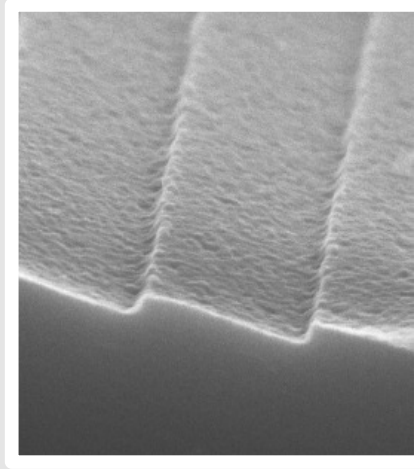

Figure 5: SEM pictures of symmetric (left figure) and asymmetric/blaze (right figure) profiles achieved directly by holographic recording at Zeiss.

Blazed gratings are generally the best choice for achieving the maximum diffraction efficiencies in a moderate wide wavelength band. However, a number of applications such as typical spectrometer systems need a broader spectral range. Here the drop in diffraction efficiency for the employed order towards the edges of the addressed spectrum limits the dynamics of the spectral sensor system. Thus a systematic approach based on a combination of interference lithography and ion beam etching has been developed at Zeiss. It provides a tuneable spectral response curve even for imaging gratings by mixing the characteristics of different blazed angles without influencing the systems spectral resolution.

Zeiss applies a new method to manufacture high quality blazed gratings from EUV to IR applications with an outstanding low level of stray light by employing a combination of holography and reactive ion etching. Therefore, our adapted recording process results in a resist mask with virtually ideal blaze structure. The etching step results in a compression or stretching of the z-component of the blaze structure whereby small surface irregularities of the resist pattern might be reduced further. The profile depth, and thus the blaze angle, of the profile can be optimized for a certain wavelength range. The main technological manufacturing steps are shown in Figure 6. The full process of the TROPOMI grating manufacturing procedure is shown in Figure 7 as an example.

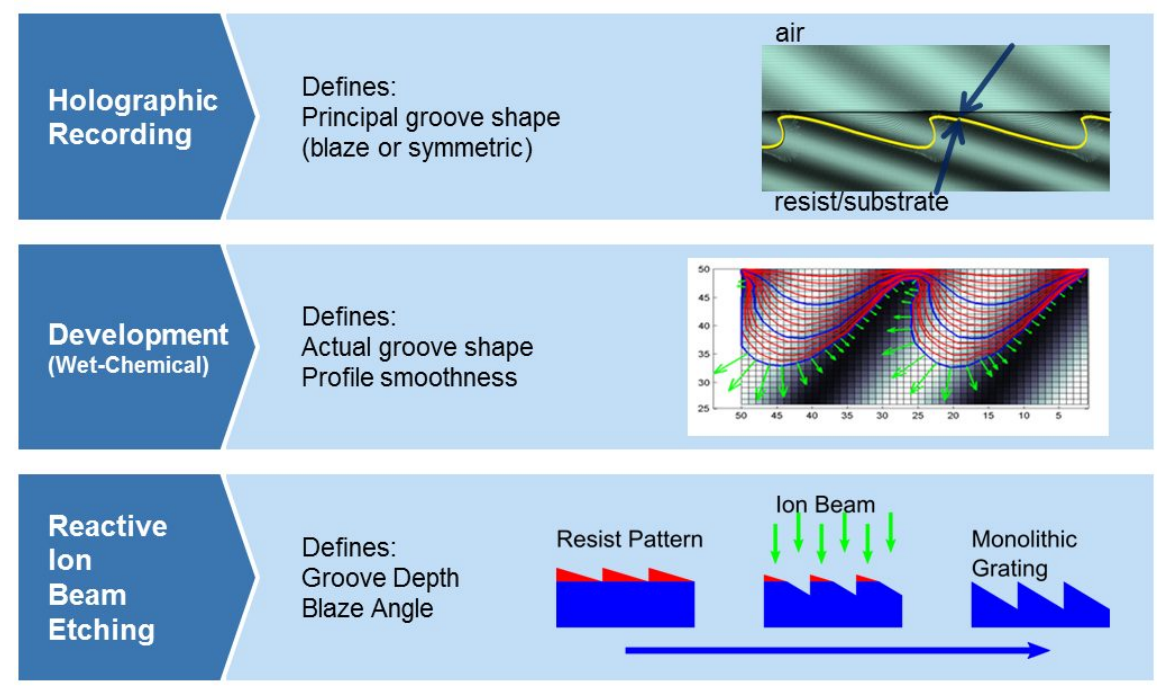

Figure 6: The main manufacturing steps for holographic grating at Zeiss. 
Zeiss uses high-precision testing methods to guarantee given specifications. In the UV wavelength range, for example, the performance of optical elements suffer in particular from any kind of geometrical errors, including roughness on the nanometer scale. Thus, a test certificate provides all requested information on the grating performance, such as slope error, micro roughness of the substrate surface, groove density, focal properties, diffraction efficiency and stray light.

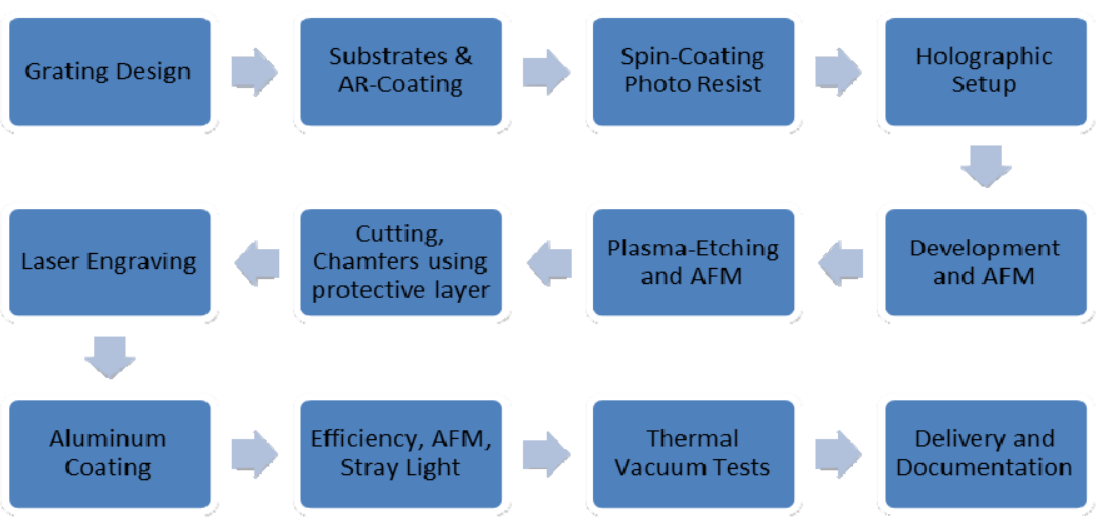

Figure 7: Full manufacturing process steps at Zeiss to secure achievement of the specifications.

The accuracy of the substrate surface or of the grating will be measured either with interferometry or, in cases where classical interferometric testing is inadequate, with a high resolution coordinate measuring device. This combination allows for measurements of nearly all substrate shapes. The slope error of the substrate surface can be measured down to 0.1 arcsec rms (depending on the actual geometry). The RMS micro roughness of the substrate surface is determined by AFM and micro interferometry. Micro interferometry allows sub-nanometer resolution of surface features with spatial frequencies between $1 \mathrm{~mm}$ and a few microns. For roughness detection on smaller level of spatial frequency (several microns down to $10 \mathrm{~nm}$ ) an AFM is used. The groove density can be determined by AFM measurements or by measuring diffraction angles with a calibrated laser set-up.

The survival of gratings exposed to environmental stress is crucial for space gratings, since service of integrated units usually is no option. Thus, the performance of the grating prior to and after environmental testing can be performed either at the Zeiss Test Center or at external institutions. Figure 8 shows, as example, the mounting of the TROPOMI gratings for the environmental tests with the testing chamber. In the efficiency and stray light measurements as well as in the cosmetic appearance, no impact of the thermal vacuum stress was observable. 


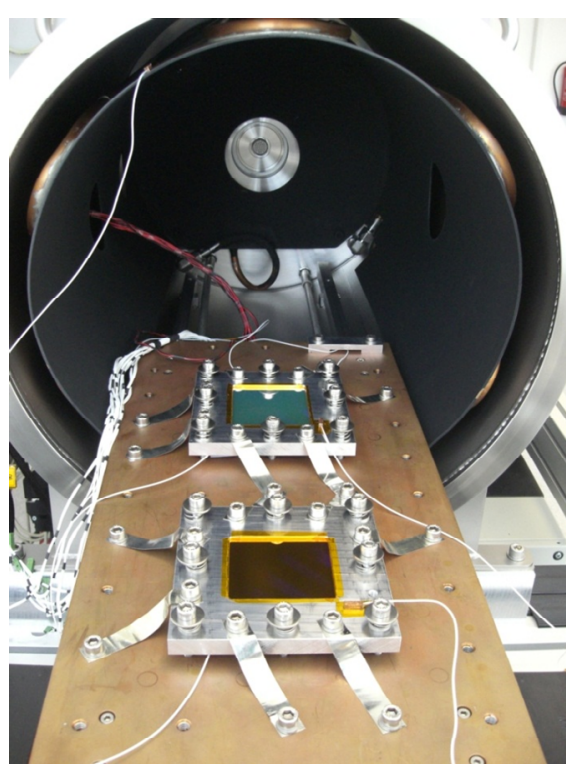

Figure 8: Mounting of the TROPOMI grating for the environmental tests.

\section{STRAYLIGHT MEASUREMENTS}

As stated above, we successfully have employed a model to predict the diffraction efficiency of realistically achievable holographically blazed gratings within the TROPOMI project [8]. Variation of different parameters in the manufacturing process allows to modify the actual groove shape intentionally, to tune the diffraction efficiency towards required values. In this context, we were able to optimize the diffraction efficiency of the gratings manufactured in the TROPOMI project to achieve very high diffraction efficiencies. Figure 9 shows the groove topography of a UVIS grating manufactured at Zeiss, whereas Figure 10 shows required (green line), the simulated (other lines) as well as the measured (points) absolute diffraction efficiency.

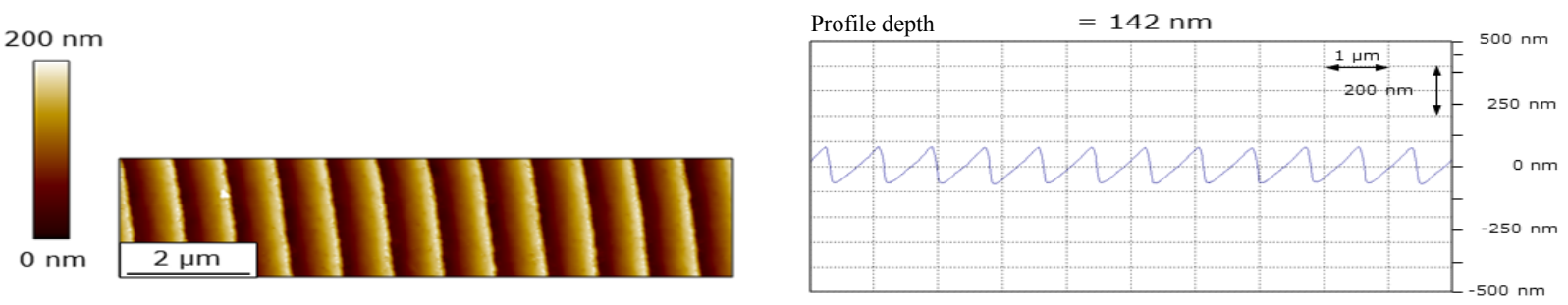

Figure 9: Topography (left) and averaged profile (right) of a TROPOMI flight grating. 


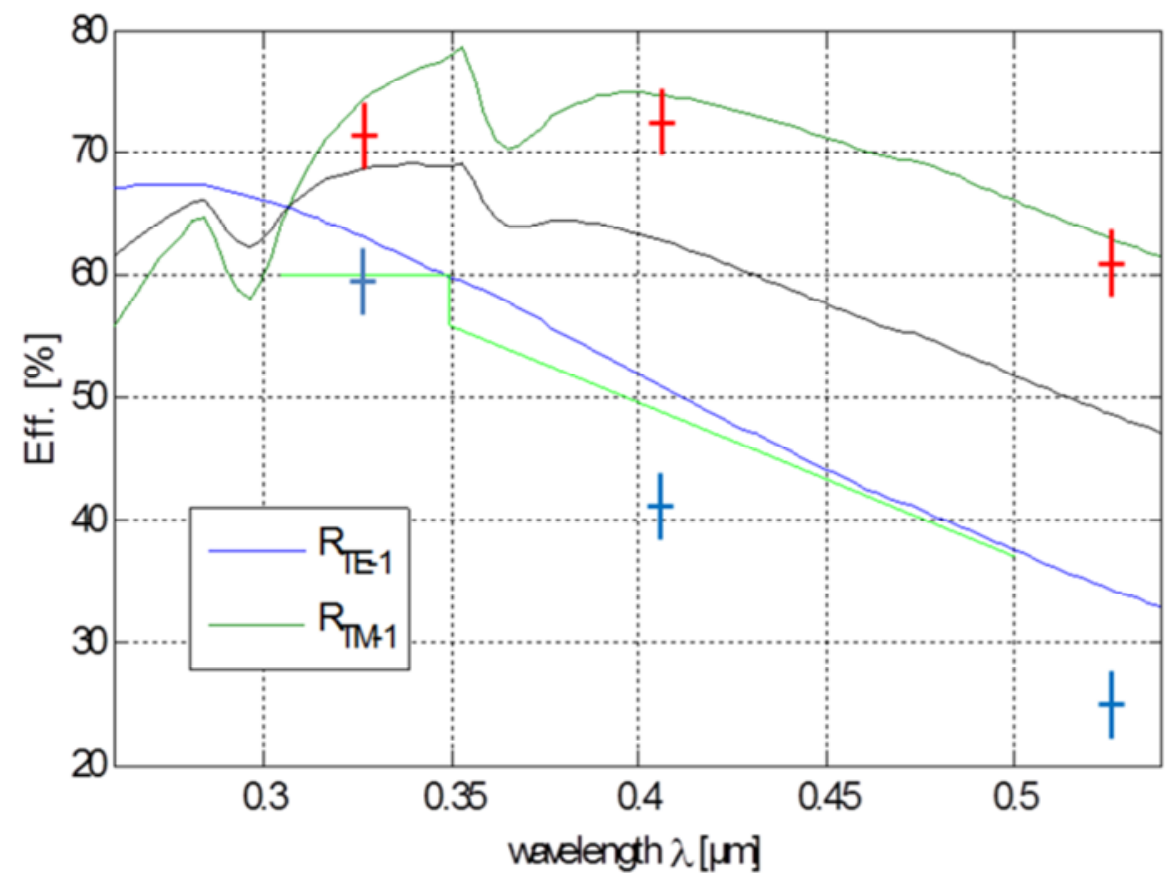

Figure 10:Required (green line) simulated TE (blue line), TM (red line) and averaged (black line) as well as measured (points) diffraction efficiency of a TROPOMI UVIS grating.

Predicting the stray light properties of a grating is much more sophisticated. Simulations here are quite sensitive to features, that might not be able to be measured separately. A good knowledge of the different process steps allows optimization of stray light by tuning process parameters. But, at the end of the manufacturing of gratings for challenging diffraction gratings, measurement of the actual stray light level, preferably with extremely high precisions-, has to be performed. Here Zeiss relies on internal as well as external measurement capabilities.

For measuring actual stray light of optical components, one of the best addresses is TNO in Delft. There, a selfdeveloped setup with extreme precision is available. A short sketch with a few properties is shown in Figure 11.

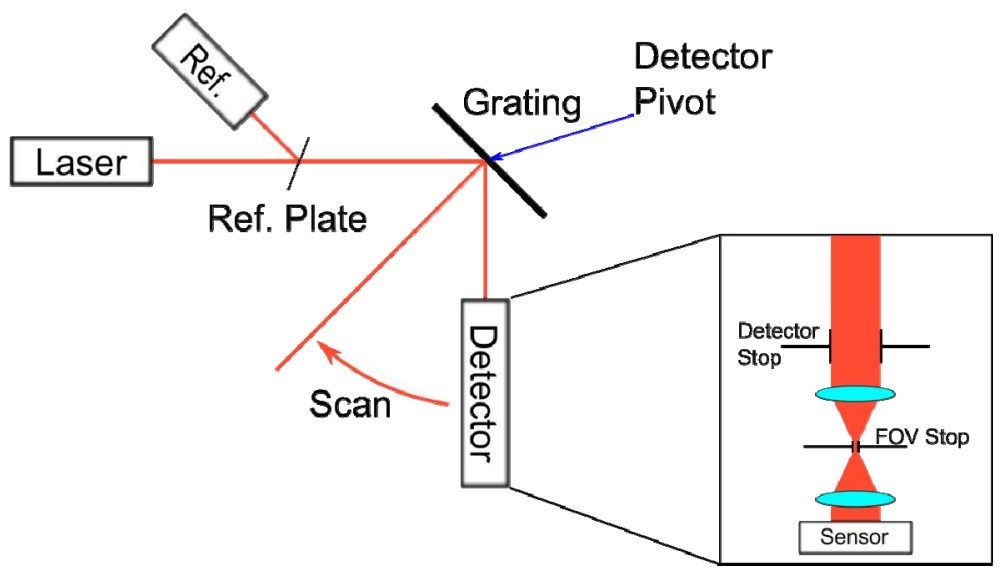

Figure 11: Sketch of setup at TNO (Delft) for measurement of stray light of optical components. Typically a laser with $532 \mathrm{~nm}$ wavelength is applied. Its 1/e-gaussian diameter on the sample is $2.25 \mathrm{~mm}$. With the FOV stop as FOV of $0.83^{\circ}$ is realized.

The stray light properties of the TROPOMI gratings were measured at TNO, using the setup described above. The bidirectional scatter distribution function (BSDF) of a TROPOMI UVIS grating is shown in Figure 12. Besides the data 
of this grating, the results of a wafer, as a perfect flat, of a reference mirror, as the typically achievable level for glass optics, as well as the typically achieved level of ruled gratings is shown there. As can be seen, between two orders a stray light level of about $10^{-7}$ was achieved. Additionally, this level is quite near to the reference mirror, pronouncing the low stray light level of the grating.

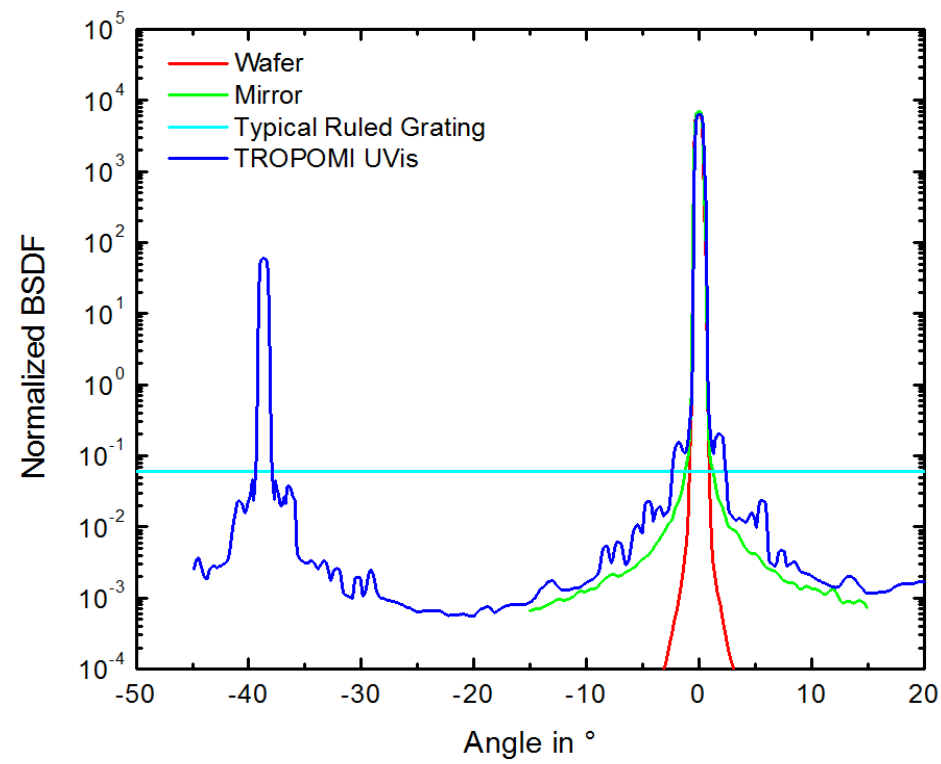

Figure 12: Stray light level of a wafer, a reference mirror and a TROPOMI UVIS grating measured at TNO. Additionally, the typical level of ruled gratings is shown.

\section{CONCLUSIONS}

In the frame of the TROPOMI project, Zeiss has proven capable of the manufacturing of high quality gratings suited for demanding space instruments. The gratings are capable of withstanding harsh thermal vacuum conditions and demonstrate extremely low stray light levels, achievable at Zeiss, while having excellent diffraction efficiency performance. Through a long history of challenging development of innovative manufacturing techniques for various projects from space telescopes over microscopy to optics for micro lithography, Zeiss has gained expertise in numerous fields of optics fabrication. In the field of microstructured optics, the company is specialized in the fabrication of custom designed gratings of highest quality covering the spectral ranges from XUV to FIR. This is based on holographic exposure and the expertise in the dry etching processes, sustained by the full manufacturing network of Zeiss (optical design, substrate fabrication, coating technologies, climate testing, optical shop testing, quality assurance). Indeed Zeiss gratings fulfill the challenging demands of todays and future operation in harsh environments such as space missions, laser pulse compressors and spectroscopic applications.

\section{REFERENCES}

[1] http://www.tropomi.eu/TROPOMI/Home.html

[2] http://www.zeiss.de/gratings

[3] http://www.zeiss.de/spectral

[4] http://czjena.zeiss.com/carl-zeiss-jena/en_de/capabilities/optical-omponents/microoptics.html 
[5] Alexandre Gatto, "Microstructured optics for high performance optical systems", Proceedings of SPIE Vol. $8613,86130 \mathrm{U}(2013)$

[6] Oliver Sandfuchs, Matthias Burkhardt, Reinhard Steiner, Alexandre Gatto, Robert Brunner, “ Holographically microstructured gratings for high-performance spectrometers", DGaO Proceedings 2011, http://www.dgaoproceedings.de, ISSN: 1614-8436, urn:nbn:de:0287-2011-B027-2

[7] Jacqueline Maass, Oliver Sandfuchs, Alexandre Gatto, et al., "Talbot-carpets of periodic and quasi-periodic close-packed 2D mask structures calculated by a modified chirp-z-algorithm", Proceedings of SPIE Vol. 8428, 84281L (2012)

[8] Oliver Sandfuchs, Alexander Pesch, Robert Brunner, "Rigorous modeling of dielectric and metallic blaze gratings in the intermediate structure regime", SPIE Proceedings Vol. 6675 (2007)

[9] O. Sandfuchs, et al., Modelling adapted to manufacturing aspects of holographic grating structures, Journal of the European Optical Society - Rapid Publications 6, 11006 (2011)

[10]R. Brunner et. al."Diffractive refractive hybrid microscope objective for $193 \mathrm{~nm}$ inspection systems", Proc. SPIE 5177, 9-15 (2003).

[11]R. Brunner, et. al. , "New solutions to realize complex optical systems by a combination of diffractive and refractive optical components", Proc. SPIE 5183, 47-55 (2003).

[12] M. Burkhardt et al., "Functional integrated optical elements for beam shaping with coherence crambling property, realized by interference lithography” Appl. Optics 46, 7061-7067, (2007). 